\title{
Local recurrence and occult neoplastic cells in the extranodal fat of dissected lymph nodes in patients with curatively resected primary colorectal cancer
}

\author{
MASAYA MUKAI $^{1}$, MASATO NAKAMURA ${ }^{2}$, KYOKO KISHIMA $^{1}$, HIROMI NINOMIYA $^{3}$, \\ NOZOMI NOMURA ${ }^{2}$, HISAYOSHI SATO ${ }^{2}$, NOBUAKI KATO ${ }^{2}$, TOMOHISA MACHIDA $^{2}$, \\ HISAO NAKASAKI ${ }^{3}$ and HIROYASU MAKUUCHI ${ }^{4}$
}

\begin{abstract}
Departments of ${ }^{1}$ Surgery, ${ }^{2}$ Pathology, Tokai University Hachioji Hospital, Ishikawa-cho 1838, Hachioji, Tokyo 192-0032;
${ }^{3}$ Department of Surgery, Tokai University Oiso Hospital, Gakyou 21-1, Oiso, Kanagawa 259-0198; ${ }^{4}$ Department

of Surgery, Tokai University School of Medicine, Bohseidai, Isehara, Kanagawa 259-1193, Japan
\end{abstract}

Received February 5, 2007; Accepted March 19, 2007

\begin{abstract}
This study was designed to examine the relationship between occult neoplastic cells (ONCs) inside and outside harvested lymph nodes (intranodal/extranodal ONCs) and local recurrence in 30 patients who underwent curative resection of primary colorectal cancer. Among 10 patients with colon cancer (Dukes' $\mathrm{A}=1$, Dukes' $\mathrm{B}=6$ and Dukes' $\mathrm{C}=3$ ), intranodal ONCs were positive in 1 patient $(10.0 \%)$ and negative in 9 patients $(90.0 \%)$, while extranodal ONCs were negative in all 10 patients $(100.0 \%)$. There were no significant differences between the detection of intranodal or extranodal ONCs. Among 20 patients with rectal cancer (Dukes' $\mathrm{A}=4$, Dukes' $\mathrm{B}=2$ and Dukes' $\mathrm{C}=14$ ), intranodal ONCs were positive in $5(25.0 \%)$ and negative in $15(75.0 \%)$, while extranodal ONCs were positive in $3(15.0 \%)$ and negative in $17(85.0 \%)$. There were no significant differences between the detection of intranodal or extranodal ONCs. These results suggest that patients with rectal cancer and extranodal ONCs should be followed-up carefully as a high-risk group for pelvic local recurrence. However, the prevalence of extranodal and intranodal ONCs was almost similar.
\end{abstract}

\section{Introduction}

It is thought that metastasis of colorectal cancer to the liver or lungs occurs in patients who have undergone radical curative

Correspondence to: Dr Masaya Mukai, Department of Surgery, Tokai University Hachioji Hospital, Ishikawa-cho 1838, Hachioji, Tokyo 192-0032, Japan

E-mail: mukai.masaya@hachioji-hosp.tokai.ac.jp

Abbreviations: LNs, lymph nodes; ONCs, occult neoplastic cells; ITCs, isolated tumor cells

Key words: colorectal cancer, local recurrence, occult neoplastic cells, extranodal tumor deposit, cytokeratin immunohistochemistry, isolated tumor cells resection when micrometastases or free cancer cells circulating during the perioperative period avoid the host immunological response and survive to proliferate in distant organs (1-4). Free cancer cells are mentioned as isolated tumor cells (ITCs) under breast cancer in the sixth version of the TNM classification published in 2002 (5). At the St. Gallen meeting in 2005 , ITCs were also defined as cells/clusters with a diameter $\leq 0.2 \mathrm{~mm}$ that had not penetrated the walls of vessels or lymphatics (Viale G, 9th International Conference on Primary Therapy of Early Breast Cancer, St.Gallen: abs. S12, 2005). Larger clusters of floating cancer cells are also observed occasionally, but the significance of such cell clusters remains unclear. On the other hand, many studies have shown that free cancer cells can be detected in lymph node (LN) sinuses distant from the primary tumor by cytokeratin immunohistochemical staining, and these are known as occult neoplastic cells (ONCs) and are closely related to tumor recurrence/metastasis with various types of cancer (6$9)$. ONCs can be counted after immunohistochemical staining by a simple method (patent pending since 2002, Japan), which reveals a small number of malignant cells trapped in the lymph nodes $(2,3)$. Solitary ONCs floating in LN sinuses far from the primary tumor can only be detected by cytokeratin immunostaining and ONCs are defined to also include clusters of $\leq 10$ cells, while malignant microaggregates contain $>10$ cells $(2,3,10)$. ONC clusters have the potential to cause distant metastasis/recurrence in any organ, and thus should be differentiated from micrometastases $(0.2 \mathrm{~mm}<\mathrm{MM} \leq 2 \mathrm{~mm})$ anchored in LNs or from ITCs $(\leq 0.2 \mathrm{~mm})$, because ONCs seem to be more malignant and act as occult systemic metastases (10-12).

Low rectal cancer is generally known to recur in the perineal region after resection or at the anastomotic site or locally in the presacral region after low anterior resection and/or Miles' operation (13). The 5-year survival rate of stage II/III rectal cancer patients is about $80-85 \%$ and $60-65 \%$, respectively. The postoperative distant metastasis/recurrence rate in patients with stage III rectal cancer is as high as 35$40 \%$ and the postoperative local pelvic recurrence rate of 


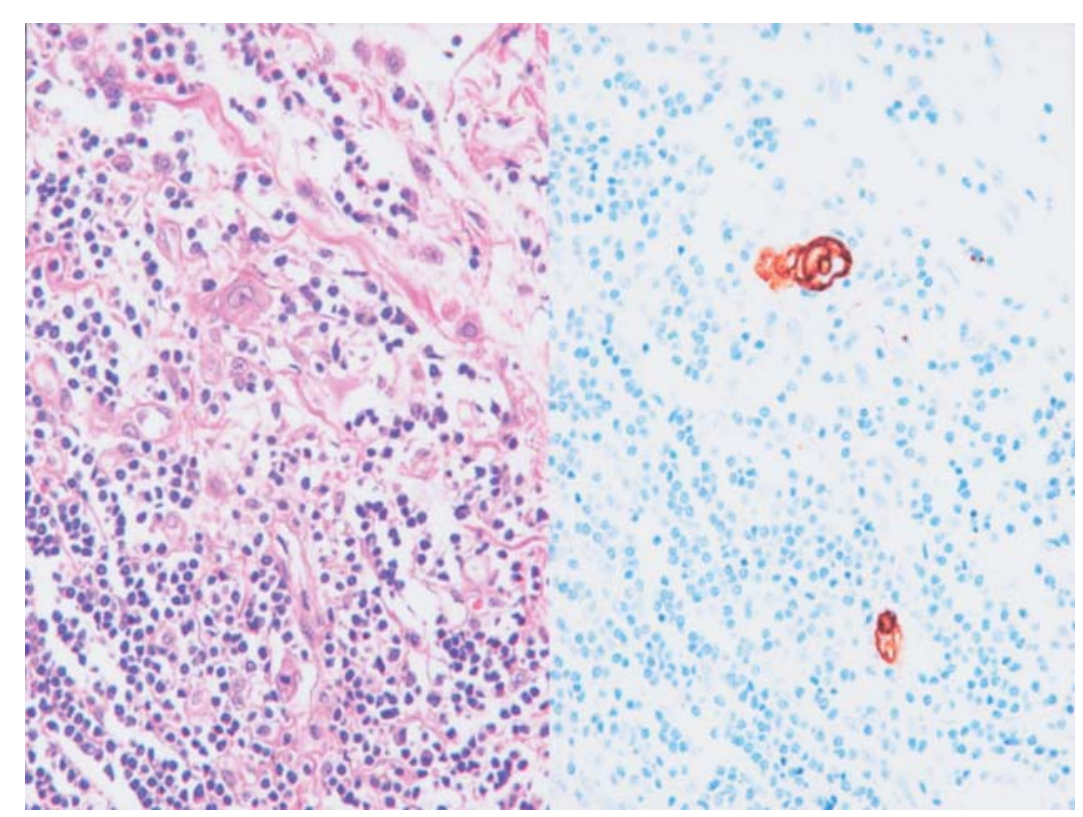

Figure 1. Immunohistochemical staining of intranodal occult neoplastic cells (intranodal ONCs) in the lymph node sinuses of a patient with stage II/ Dukes' B rectal cancer from the local recurrence group (left: H\&E stain, $x 400$; right: cytokeratin stain, $x 400$ ).

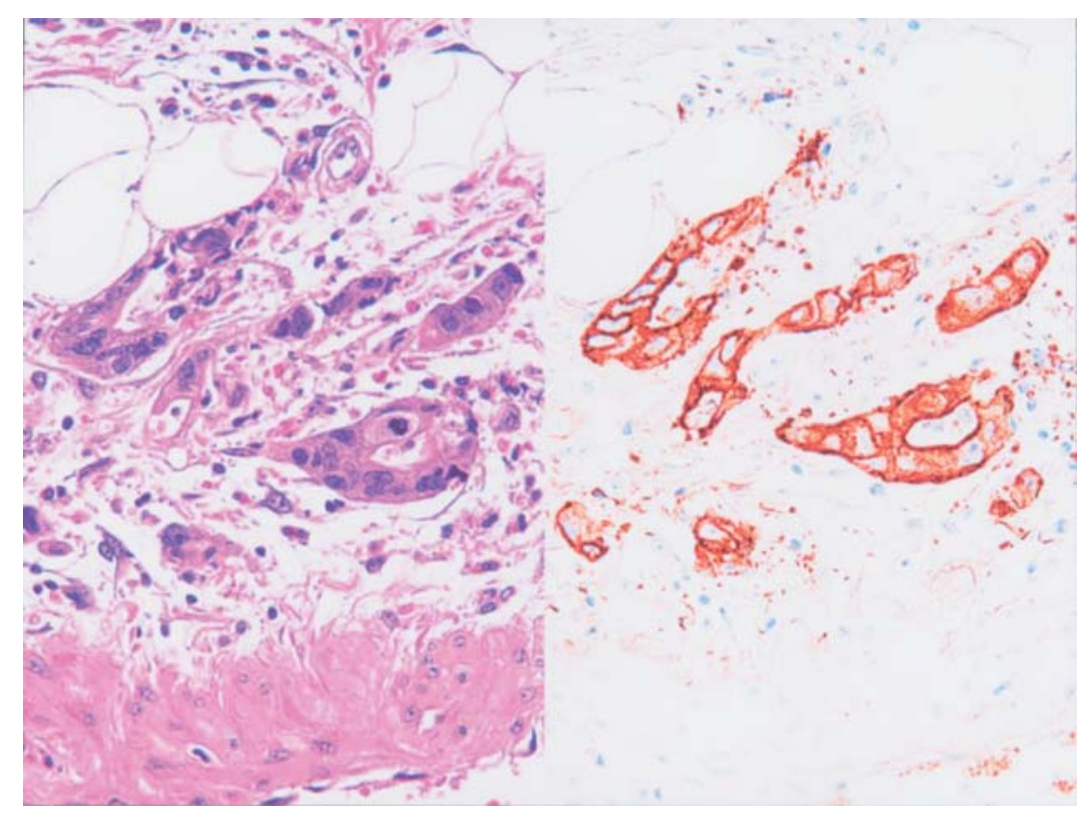

Figure 2. Immunohistochemical staining of extranodal occult neoplastic cells (extranodal ONCs) and extranodal ONC clusters in the perinodal fat around the dissected lymph node of a patient with stage II/Dukes' B rectal cancer from the local recurrence group (left: H\&E stain, x400; right: cytokeratin stain, x400).

patients with stage II/III rectal cancer is about $15-20 \%$ (1416), indicating that rectal cancer has a characteristic pattern of recurrence unlike colon cancer that is associated with hematogenous metastasis to the liver or lungs (17-19). The risk factors for postoperative local recurrence of lower rectal cancer include: i) an inadequate resection margin of $<2 \mathrm{~cm}$, ii) seeding of cancer cells by iatrogenic bowel perforation or postoperative suture failure, iii) implantation of viable cancer cells in the peritoneal cavity by surgical procedures such as mechanical anastomosis, and iv) unrecognized microscopic tumor extension including undetectable microscopic lymph node metastasis or microscopic tumor deposits in the pelvic fat $(13,20,21)$. With respect to microscopic tumor deposits in the pelvic fat, a recent study showed that cytokeratin-positive ONC clusters can be detected in the perinodal fat around dissected lymph nodes (extranodal ONCs) (22).

Although there have been many reports about the relationship between intranodal ONCs and distant metastasis/ recurrence of primary colorectal cancer, there is no report on a clinicopathological examination of the relationship between local recurrence of colorectal cancer and extranodal ONCs. Accordingly, the present study was designed to examine the relationship between intranodal/extranodal ONCs detected using cytokeratin immunohistochemistry and local recurrence of primary colorectal cancer in patients who had undergone curative resection. 
Table I. Intranodal occult neoplastic cells (ONCs) in the lymph node sinuses and extranodal ONCs in the dissected perinodal fat of 30 patients with local reccurence of primary colorectal cancer.

\begin{tabular}{|c|c|c|c|c|}
\hline \multirow{3}{*}{$\begin{array}{l}\text { Local recurrence } \\
\text { Total } 30 \text { cases }\end{array}$} & \multicolumn{4}{|c|}{ Cytokeratin immunostaining $[(-)<3$ ONCs $\leq(+)]$} \\
\hline & \multicolumn{2}{|c|}{ Intranodal ONCs $(\%)$} & \multicolumn{2}{|c|}{ Extranodal ONCs $(\%)$} \\
\hline & $(+)$ & $(-)$ & $(+)$ & $(-)$ \\
\hline Colon $(\mathrm{n}=10)$ & $10.0(1 / 10)$ & $90.0 \quad(9 / 10)$ & $0.0(0 / 10)$ & $100.0(10 / 10)$ \\
\hline Rectum $(n=20)$ & $25.0(5 / 20)$ & $75.0(15 / 20)$ & $15.0(3 / 20)$ & $85.0(17 / 20)$ \\
\hline
\end{tabular}

In the colon cancer group $(n=10)$ and the rectal cancer group $(n=20)$, the expression was classified as positive when $\geq 3$ ONCs were detected in each type of ONCs. ONCs, occult neoplastic cells.

\section{Materials and methods}

Among patients who underwent curative resection of primary colorectal cancer from 1987 to 2001, 39 patients who had limited recurrence at the primary tumor site or nearby on postoperative US/CT were defined as having local recurrence. Of these 39 patients, 30 (5 with Dukes' A, 8 with Dukes' B and 17 with Dukes' $\mathrm{C}$ cancer) who met the following criteria were investigated: i) accurate and complete medical records were available and they could be followed to assess survival time, and ii) at least 10 histologically negative lymph nodes as determined by routine H\&E staining were collected during surgery. The 30 patients were divided into 10 with colon cancer (1 with Dukes' A, 6 with Dukes' B and 3 with Dukes' C cancer) and 20 with rectal cancer (4 with Dukes' A, 2 with Dukes' B and 14 with Dukes' $\mathrm{C}$ cancer). The detection rates of intranodal and extranodal ONCs were calculated for each and compared between them.

Immunohistochemical staining of harvested LNs was performed by a routine indirect immunoperoxidase method using a monoclonal anti-cytokeratin antibody (No. 760-2135; pan-keratin, Ventana Japan, Japan) (23-26). A total of 10 lymph nodes that were determined to be negative for metastasis by routine $H \& E$ staining were randomly selected from among the dissected samples. Accordingly, a total of 300 nodes were selected from the 30 patients for examination. Serial sections of each lymph node were stained with H\&E or the above-mentioned antibody to determine the positive rate and positive cell count for each stain. Tumor cells and/or cell nests attached to the lymph node tissues were excluded, and only free neoplastic cells in the lymph node sinuses (intranodal ONCs) or in the extranodal fat around the dissected LNs (extranodal ONCs) were counted (Figs. 1 and 2). Immunostaining for cytokeratin was assessed as positive if there were 3 or more cytokeratin-positive cells (2-4).

Statistical analysis. The $\chi^{2}$ test was used to examine the significance of differences between the recurrence and nonrecurrence groups. A P-value of $<0.05$ was considered to indicate a significant difference in all analyses, and SPSS $13.0 \mathrm{~J}$ software (SPSS Japan, Inc., Tokyo, Japan) was used.

\section{Results}

Intranodal ONCs were observed in 1 of the 10 patients with colon cancer (1 with Dukes' A, 6 with Dukes' B and 3 with Dukes' C cancer), so intranodal ONCs were positive in $10.0 \%$ and negative in $90 \%$ (Table I). None of the 10 patients with colon cancer had extranodal ONCs, so extranodal ONCs were positive in $0.0 \%$ and negative in $100 \%$ (Table I). There were no significant differences between the detection rates of intranodal and extranodal ONCs in the patients with colon cancer (Table I). Intranodal ONCs were observed in five of the 20 patients with rectal cancer (4 with Dukes' A, 2 with Dukes' B and 14 with Dukes' C cancer), so intranodal ONCs were positive in $25.0 \%$ and negative in $75 \%$ (Table I). Extranodal ONCs were observed in 3 of the 20 patients, so extranodal ONCs were positive in $15.0 \%$ and negative in $85.0 \%$ (Table I). There were no significant differences between the detection rates of intranodal and extranodal ONCs in the patients with rectal cancer (Table I). No significant differences were noted for the detection rates of either intranodal or extranodal ONCs between the colon and rectal cancer groups (Table I).

\section{Discussion}

About $15-20 \%$ of patients with resected rectal cancer develop local recurrence in the pelvic cavity or presacral region and a certain percentage or stage I-III rectal cancer recurs even after curative resection (13-16). The main causes of recurrence include insufficient separation between the distal bowel stump and the mesorectum or an inadequate surgical margin for the distal stump. However, a distal margin of at least $2 \mathrm{~cm}$ which is a requirement for curative resection of colorectal cancer according to the Japan Colorectal Cancer Society Rules, is secured in most patients who undergo low anterior resection $(1,13,14)$. It has been reported that extranodal tumor deposits in the resected fat of patients with breast, esophageal, or lung cancer are closely related to metastasis/recurrence (27-29). These tumor deposits are large enough to be easily detected by routine $\mathrm{H} \& \mathrm{E}$ staining in patients with various types of cancer, including rectal cancer. They are often detected in resected fat tissue during the assessment of perineural invasion or tumor spread at the distal margin of lymph node dissection 
(30-35). Although the presence of ONCs (single tumor cells) can only be confirmed by immunohistochemical staining, it indicates a high risk of distant metastasis/recurrence for stage I/II N0-local disease after curative resection (2-4). Particularly, ONC clusters $(\leq 10 \mathrm{ONCs})$ or malignant micro-aggregates ( $>10$ ONCs) are more likely to survive in a new microenvironment and proliferate to cause micro-metastasis and pelvic local recurrence (10-12,22). Therefore, detection of extranodal ONCs in the fat surrounding dissected lymph nodes is considered to indicate a high risk for local recurrence. Low anterior anastomosis should be carefully performed even for stage I rectal cancer when these cells are detected (22).

Many studies have shown that intranodal ONCs indicate the systemic dissemination of tumor cells more accurately than ly or $\mathrm{v}$ in the primary tumor (7-12). ONCs are different from ly/v because they are detected at a site remote from the primary tumor, irrespective of being intranodal or extranodal. In the present study, many clusters of extranodal ONCs were detected in the resected fat from around the distal stump in all three patients with rectal cancer (stage I-III). Of these three patients, however, only one patient with stage III cancer had both intranodal and extranodal ONC clusters. Intranodal ONCs were detected by examination of 10 cancer-negative nodes, as determined by routine $\mathrm{H} \& \mathrm{E}$ staining, that were removed during D2 or higher dissection. In the present study, lymph node tissue samples and the surrounding residual fat were immunostained to detect extranodal ONCs. Since it is impossible to efficiently investigate all the collected fat for extranodal ONCs, it is necessary to prepare a tissue sample of about $1 \mathrm{~cm}^{3}$ with a capsule of fat when resecting each lymph node. For the detection of extranodal ONCs, it may be necessary to include careful sampling of fat from the bilateral anterior sacral and urinary bladder base regions resected during surgery in patients with rectal cancer.

It has been reported that the rectum should be thoroughly irrigated before anastomosis during surgery for lower rectal cancer to prevent small tumor cell masses from the primary lesion being implanted in the residual rectum $(17,20,21)$. Extranodal ONC clusters may reach the anterior sacral surface or pelvic floor when the mesorectal lymphatics/veins lateral to the prostate gland or the bilateral inguinal regions are separated during peritoneal reflection or later procedures. Extranodal ONC clusters observed in the present study were detected inside the perinodal fat in almost all the cases and had a clearly different morphology from contaminating tumor cells that entered the sections during processing of the resected lymph node tissues. To prevent ONC clusters from implanting into the soft tissues of the pelvic floor, routine radiotherapy is considered effective. When low rectal anastomosis is performed, however, abdominal irrigation with anticancer drugs before anastomosis may be safer for the tissue near the anastomotic site and more effective for the deep pelvic tissues than radiotherapy.

There was no difference between the detection rates of intranodal/extranodal ONCs in patients with rectal cancer, probably because of the small number of patients. However, it may be necessary to select patients with a high risk of the local recurrence of rectal cancer by examining both intranodal and extranodal ONCs. More detailed investigation is warranted to determine whether additional resection by
Miles' operation to remove additional tissue on the anal side or a combination of radiotherapy and systemic consolidation chemotherapy is required for stage I/II N0-local disease when extranodal ONC clusters are detected postoperatively in the resected fat tissues.

\section{Acknowledgements}

This study was supported by grants from the Occult Neoplastic Cells Research and Study Group (No. 2006-5048; Tokai University Hachioji Hospital, Hachioji, Tokyo, Japan) and the Research and Study Program of Tokai University Educational System General Research Organization (No. 2005-41; Tokai University Hospital, Isehara, Kanagawa, Japan).

\section{References}

1. Mukai M, Ito I, Mukoyama S, Tajima T, Saito Y, Nakasaki H, Sato $\mathrm{S}$ and Makuuchi $\mathrm{H}$ : Improvement of 10 -year survival by Japanese radical lymph node dissection in patients with Dukes' B and C colorectal cancer: a 17-year retrospective study. Oncol Rep 10: 927-934, 2003.

2. Mukai M, Sato S, Komatsu N, Nishida T, Shiba K, Ito I, Nakasaki $\mathrm{H}$ and Makuuchi $\mathrm{H}$ : Correlation between occult neoplastic cells in the lymph node sinuses and recurrence in patients with Dukes' C colorectal cancer. Oncol Rep 10: 11651169, 2003.

3. Mukai M, Sato S, Komatsu N, Nishida T, Shiba K, Ito I, Nakasaki $\mathrm{H}$ and Makuuchi H: Correlation between occult neoplastic cells in the lymph node sinuses and recurrence in patients with curatively resected Dukes' B colorectal cancer. Oncol Rep 10: 1177-1181, 2003.

4. Mukai M, Sato S, Nishida T, Komatsu N, Shiba K, Nakasaki H and Makuuchi H: Selection criteria for high-risk and low-risk groups of recurrence and metastasis in patients with primary colorectal cancer. Oncol Rep 10: 1753-1758, 2003.

5. TNM classification of malignant tumours. 6th edition. John Wiley \& Sons Inc., NY, 2002.

6. Mukai M, Sato S, Nakasaki H, Saito Y, Nishiumi N, Iwasaki M, Tokuda Y, Ogoshi K, Inoue H and Makuuchi H: Occult neoplastic cells in the lymph node sinuses and recurrence of primary breast, lung, esophageal and gastric cancer. Oncol Rep 11: 81-84, 2004

7. Mukai M, Sato S, Tajima T, Kimura T, Komatsu N, Ninomiya H, Nakasaki H, Ogoshi K and Makuuchi $\mathrm{H}$ : Predicting recurrence and metastasis of stage II/Dukes' B colorectal cancer without lymph node metastasis. Oncol Rep 12: 1127-1130, 2004.

8. Mukai M, Sato S, Tajima T, Kimura T, Komatsu N, Ninomiya H, Nakasaki H, Ogoshi K and Makuuchi H. Predicting recurrence and metastasis of stage III/Dukes' C colorectal cancer with lymph node metastasis. Oncol Rep 12: 1301-1304, 2004

9. Mukai M, Sato S, Tajima T, Kimura T, Komatsu N, Ninomiya H, Nakasaki H, Ogoshi K and Makuuchi $\mathrm{H}$ : Predicting recurrence and metastasis of Dukes' A primary colorectal cancer with or without proper muscle invasion. Oncol Rep 12: 1305-1308, 2004.

10. Mukai M: Occult neoplastic cells and malignant microaggregates in lymph node sinuses: review and hypothesis. Oncol Rep 14: 173-175, 2005.

11. Mukai M, Sato S, Ninomiya H, Wakui K, Komatsu N, Tsuchiya K, Tajima T, Nakasaki $\mathrm{H}$ and Makuuchi $\mathrm{H}$ : Prospective study on the recurrence/metastasis of stage II/III colorectal and gastric cancer associated with occult neoplastic cells in lymph node sinuses: three-year interim results. Oncol Rep 16: 405-410, 2006.

12. Mukai M, Oida Y, Tajima T, Kishima K, Ninomiya H, Sato S, Nakamura M, Nakasaki H and Makuuchi H: Alternating hepatic arterial infusion and systemic chemotherapy for stage IV colorectal cancer with synchronous liver metastasis. Oncol Rep 16: 865-870, 2006.

13. Knack \& Pit falls: Surgery of the colon, rectum and anus. 2nd edition. Bunkoudou Co., Ltd., Tokyo, 2004

14. Guide line of large Bowel Cancer in Japan. Japanese Society for Cancer of the Colon and Rectum, Tokyo, 2005. 
15. Multi-Institutional Registry of Large Bowel Cancer in Japan. Vol. 23. Cases treated in 1994. Japanese Society for Cancer of the Colon and Rectum, Tokyo, 2002.

16. Journal of Health and Welfare Statistics: Health and Welfare Statistics Association. Tokyo, 52: 45-47, 2005.

17. Hurst PA, Prout WG, Kelly JM, Bannister JJ and Walker RT: Local recurrence after low anterior resection using the staple gun. Br J Surg 69: 275-276, 1982.

18. Tomoda $\mathrm{H}$ and Furusawa $\mathrm{M}$ : Local recurrence after curative anterior resection for carcinoma of the rectum. Jpn J Surg 16: 377-379, 1986.

19. Suzuki K, Muto T and Sawada T: Prevention of local recurrence by extended lymphadenectomy for rectal cancer. Jpn J Surg 25: 795-801, 1995.

20. Syfan J, Averbuch F, Koltun L and Benyamin N: Effect of rectal stump washout on the presence of free malignant cells in the rectum during anterior resection for rectal cancer. Dis Colon Rectum 43: 1710-1712, 2000.

21. Terzi C, Ünek T, Sagol O, Yilmaz T, Füzün M, Sökmen S, Ergör $G$ and Küpelioglu A: Is rectal washout necessary in anterior resection for rectal cancer? A prospective clinical study. World J Surg 30: 233-241, 2006.

22. Hoshikawa T, Mukai M, Oida Y, Tajima T, Morikawa G, Nakamura T, Motojyuku M, Nakamura $M$ and Makuuchi $H$ : Pelvic recurrence after Miles' operation for anastomotic recurrence in a patient with stage I rectal cancer invading the proper muscle layer: Case report. Oncol Rep 17: 743-746, 2007.

23. Nakane PK and Pierce GB: Enzyme-labeled antibodies: preparation and application for localization of antigens. J Histochem Cytochem 14: 929-931, 1966.

24. Nakane PK and Pierce GB: Enzyme-labeled antibody for the light and electron microscopic localization of tissue antigens. J Cell Biol 33: 307-318, 1967

25. Okabe H, Tsujimoto $H$ and Fukushima M: Preparation of the antibodies against recombinant human thymidylate synthase for the detection of its intratumoral levels and application to sensitivity-study of 5-fluorouracil. Oncol Rep 4: 685-690, 1997.
26. Kamoshida S, Shiogama K, Matsuoka H, Matsuyama A, Shimomura R, Inada K, Maruta M and Tsutsumi Y: Immunohistochemical demonstration of dihydropyrimidine dehydrogenase in normal and cancerous tissues. Acta Histochem Cytochem 36: 471-479, 2003.

27. Mambo NC and Gallager HS: Carcinoma of the breast: the prognostic significance of extranodal extension of axillary disease. Cancer 39: 2280-2285, 1977.

28. Suemasu K and Naruke T: Prognostic significance of extranodal cancer invasion of mediastinal lymph nodes in lung cancer. Jpn J Clin Oncol 12: 207-212, 1982.

29. Watanabe H, Kato H, Tachimori Y, Yamaguchi H and Itabashi M: Characteristics of the spread pattern for esophageal carcinoma (in Japanese with English abstract). Jpn J Thorac Surg 42: 682-689, 1989.

30. Ueno $\mathrm{H}$ and Mochizuki $\mathrm{H}$ : Clinical significance of extra bowel skipped cancer infiltration in rectal cancer. Jpn J Surg 27: 617-622, 1997.

31. Ueno H, Mochizuki $\mathrm{H}$ and Tamakuma S: Prognostic significance of extranodal microscopic foci discontinuous with primary lesion in rectal cancer. Dis Colon Rectum 41: 55-61, 1998.

32. Singh AK, Myerson RJ, Birnbaum EH, Fleshman JW, Kodner IJ, Lokett MA and Read TE: Outcome of patients with rectal adenocarcinoma and localized pelvic non-nodal matastatic foci. Dis Colon Rectum 43: 1217-1221, 2000

33. Ueno H, Hase K and Mochizuki H: Criteria for extramural perineural invasion as a prognostic factor in rectal cancer. $\mathrm{Br} \mathbf{J}$ Surg 88: 994-1000, 2001.

34. Ishikawa K, Hashiguchi Y, Mochizuki H, Ozeki Y and Ueno H: Extranodal cancer deposit at the primary tumor site and the number of pulmonary lesions are useful prognostic factors after surgery for colorectal lung metastases. Dis Colon Rectum 46: 629-636, 2003.

35. Prabhudesai A, Arif S, Finlayson CJ and Kumar D: Impact of microscopic extranodal tumor deposits on the outcome of patients with rectal cancer. Dis Colon Rectum 46: 1531-1537, 2003. 